$9^{\text {th }}$ International Workshop on Innovative Simulation for Healthcare

$17^{\text {th }}$ International Multidisciplinary Modeling \& Simulation Multiconference

ISSN 2724-0371 ISBN 978-88-85741-50-8 (C) 2020 The Authors.

DOI: $10.46354 /$ i3m.2020.iwish.011

\title{
Electronic health bubble: the caregiving services at the time of the covid-19 outbreak
}

\author{
Marco Frascio ${ }^{1 *}$, Cesare Stabilini ${ }^{1}$, Franco Borasi $^{2}$, Luca Bordignon $^{2}$, Giuseppe \\ Molinari ${ }^{3}$, Vera Novak $^{4}$, and Rosario Fornaro ${ }^{1}$
}

'DISC, Dipartimento di Scienze Chirurgiche e Diagnostiche Integrate, Università di Genova - Ospedale Policlinico San Martino di Genova, Viale Benedetto XV, Genova 16131, Italy

${ }^{2}$ T.E.A Technology for Edge Applications., Via Ilva 4/4b Genova 16128. Italy

${ }^{3}$ Telemedico S.r.l. Viale Brigata Bisagno 2, Genova 16129, Italy

${ }^{4}$ Harvard University Massachusetts Hall Cambridge, MA 02138, USA

${ }^{*}$ Corresponding author. Email address: mfrascio@unige.it

\begin{abstract}
The electronic Health Bubble, from now on eHB, was conceived 24 months ago as an eHealth information and communication technology system whose aim is to help the telehealth and tele-caregiving services dedicated to elders or to patients affected by a chronic disease. Thanks to our cooperation with experts of health services and consultants for other NHSs, the eHB field of intervention has been enlarged by including all the tools, equipment, medical processes and a new social approach to cope with the second phase of the COVID-19 pandemia, after the lockdown.
\end{abstract}

Keywords: Telehealth; tele-caregiving; COVID-19 pandemia.

\section{Introduction}

The application of ICT in the medical field has obtained encouraging results since several years, about prevention (Gipponi et al. 1995), medical care (Molinari et al. 2002) and also medical teaching (Marco Frascio et al. 2009; Ferrara et al. 2016). Similarly, ICT has provided a powerful tool to improve the simulation program useful for training (Perino et al. 2015; Frascio et al. 2013) and for developing innovative response procedures during disasters, as a training strategy that combines medical skills with engineering to improve reaction capabilities and situation awareness to cope disaster crises (Frascio et al. 2019; Mandolfino, Petrillo, and Frascio 2017). The electronic Health Bubble, from now on eHB, was conceived 24 months ago as an eHealth information and communication technology system whose aim is to help the telehealth and tele-caregiving services dedicated to elders or to patients affected by a chronic disease (Frascio et al. 2018).

\section{State of the art}

eHB integrates and complements the existing telemedicine solutions by making available plug\&play interfaces and easy connection systems, safe and solid to integrate and interface with more common and used medical tools. Through a robust and reliable sensors network eHB permits to create a virtual space (the health bubble) around the patient able to control minute by minute every medical tool and drug, as well as to follow him/her in those clinically relevant

(C) 2020 The Authors. This article is an open access article distributed under the terms and conditions of the Creative Commons Attribution (CC BY-NC-ND) license (https://creativecommons.org/licenses/by-nc-nd/4.0/). 
activities that do not require medical assistance. These basic features of the eHB were and are still considered useful since the care of the patients at home, especially the elderly people, is one of the major challenges of the modern communities. For humanitarian reasons, to keep constant and hopefully improve the quality of the services provided by the NHSs; and, last but not least, for economic reasons to not make unbearable the burden of the services for the elderly on the countries balance sheet.

\section{Results and Discussion}

On March 11, 2020, the World Health Organization declared the coronavirus disease 2019 (COVID-19) outbreak as a pandemic. The response strategy included early diagnosis, patient isolation, symptomatic monitoring of contacts as well as suspected and confirmed cases, and public health quarantine. In this context, telemedicine, particularly video consultations, has been promoted and scaled up to reduce the risk of transmission. Based on a literature review, the first conceptual framework for telemedicine implementation during outbreaks was published in 2015. An updated framework for telemedicine in the COVID-19 pandemic has been defined. This framework could be applied at a large scale to improve the national public health response. All stakeholders are encouraged to address the challenges and collaborate to promote the safe and evidence-based use of telemedicine during the current pandemic and future outbreaks (Ohannessian, Duong, and Odone 2020). Thus, the COVID-19 outbreak is posing further challenges also in the sector of the telemedicine services, especially considering the home care of the elderly people (without severe diseases) and of subcritical cases. Thanks to our cooperation with experts of health services and consultants for other NHSs, the eHB field of intervention has been enlarged by including all the tools, equipment, medical processes and a new social approach to cope with the second phase of the COVID-19 pandemia, after the lockdown. While the technical issues as well as the new methodological aspects will be more or less easily dealt with by the eHB, another aspect is rapidly stepping in the limelight: the change of perspective on preparation and mitigation of the fallout of the postlockdown phase.

Three areas of intervention concerning the eHB are illustrated:

1. The new interfaces to equipment for patients moving within their homes (the 5G services and the wide spread of apps that monitor also the short-distance movements can greatly help)
2. The twofold approach adopted by the eHB to interact effectively with the elderly people and with the COVID-19 noncritical patients

3. The radical change of perspective of the eHB system in the light of the arising COVID-19 paradigm

The first two points are challenges which are dealt with by further developments of the eHB control panel (interfaces for the new equipment whatever they will be) and by means of wider collaboration with the telemedicine centers (the eHB partnership is already enlarged to a leading Italian Telemedicine Service Center). The third is much more radical and stems from what has been rightly underlined by a group of physicians at the Papa Giovanni XXIII Hospital in Bergamo: "In a pandemic, patient-centered care is inadequate and must be replaced by community centered care. Solutions for Covid-19 are required for the entire population, not only for hospitals. The catastrophe unfolding in wealthy Lombardy could happen anywhere. Clinicians at a hospital at the epicenter call for a long-term plan for the next pandemic.".

\section{Conclusions}

In the near future, the eHB will evolve towards a new community centered paradigm; and major steps will be taken to interact with the general practitioners as well as with paramedics and social entities (e.g. NPOs, care giver associations). This eHB transformation implies the development of shared services where a small group of health care personnel will deal with a large group of spread patients, living in homes located in different districts and who must be assisted with various levels of health care. Indeed, it is likely that many of the patients must only be observed and assisted without any medical treatment except for a periodic check on daily basis.

\section{Funding}

No funding to declare.

\section{References}

Ferrara, E., Ponte, S., Caputo, E., Frascio, M., Dellepiane, S. 2016. "No Title." In IST-Africa Conference, IST-Africa 2016 - 7530648.

Frascio, M., M. Gervasoni, F. Lazzara, F. Mandolfino, M. Noceti, M. Sguanci, and G. Vercelli. 2009. "New Teaching Models for the Medical School of Medicine: Comparison between Oral an Online Classes. The Experience of the Genoa School of Medicine." Journal of E-Learning and Knowledge Society 5 (3).

Frascio, M., M. Sguanci, F. Mandolfino, M. Gaudina, and E. Bellanti. 2013. "Laparoscopic Skills 
Simulator: A Gradual Structured Training Program for Acquiring Laparoscopic Abilities." In 25th European Modeling and Simulation Symposium, EMSS 2013.

Frascio, Marco, Katia Cortese, Francesco Longo, and Kirill Sinelshchikov. 2019. "Multidisciplinary Development of a Simulation of Industrial Plant Disasters Designed for Improving Safety through Training and New Procedures." International Journal of Simulation and Process Modelling. https://doi.org/10.1504/IJSPM.2019.101015.

Frascio, Marco, Margherita Gervasoni, Fabrizio Lazzara, Francesca Mandolfino, Marco Noceti, Marco Sguanci, and Gianni Vercelli. 2009. "New Teaching Models for the Medical School of Medicine: Comparison between Oral an Online Classes. The Experience of the Genoa School of Medicine." Journal of E-Learning and Knowledge Society. https://doi.org/10.20368/1971-8829/351.

Gipponi M., M Frascio, G Arnulfo, A D'Aquila. 1995. "The Computerized Outpatient Management of Subjects at Neoplastic Risk." Minerva Medica 86 (4): 149-57.

Mandolfino, F., A. Petrillo, and M. Frascio. 2017. "The Medical Point of View into a Simulation Project of Management for Safety and Security in Disasters and Emergencies of Industrial Plants (Diem-Ssp Project)." In 6th International Workshop on Innovative Simulation for Health Care, IWISH 2017, Held at the International Multidisciplinary Modeling and Simulation Multiconference, I3M 2017.

Molinari, G., G. Reboa, M. Frascio, M. Leoncini, A. Rolandi, C. Balzan, and A. Barsotti. 2002. "The Role of Telecardiology in Supporting the DecisionMaking Process of General Practitioners during the Management of Patients with Suspected Cardiac Events." Journal of Telemedicine and Telecare. https://doi.org/10.1258/1357633021937541.

Ohannessian, Robin, Tu Anh Duong, and Anna Odone. 2020. "Global Telemedicine Implementation and Integration Within Health Systems to Fight the COVID-19 Pandemic: A Call to Action." JMIR Public Health and Surveillance. https://doi.org/10.2196/18810.

Perino, E., M. Sguanci, F. Mandolfino, M. Minuto, G. Vercelli, M. Gaudina, S. Marcutti, V. Rumolo, G. Marcocci, and M. Frascio. 2015. "Low Cost Laparoscopic Training Platform: Primary Validation Process." In 8th International Workshop on Applied Modeling and Simulation, WAMS 2015. 